\title{
New physics searches with heavy flavour observables at ATLAS
}

\author{
Cristiano Alpigiani* \\ on behalf of the ATLAS Collaboration \\ University of Washington, Seattle \\ E-mail: Cristiano.Alpigiani@cern.ch
}

\begin{abstract}
Weak decays that are naturally suppressed in the Standard Model (SM), such as processes with flavour-changing neutral-currents, are perfect for indirect searches of new physics. These decays allow us to investigate higher energy ranges with respect to direct searches, thus representing a fundamental complementary tool. The measurement of the $B_{s}^{0} \rightarrow J / \psi \phi$ decay parameters, that can provide probes for physics beyond the SM, is described in this document and the results obtained with ATLAS data collected in Run 1 will be shown.
\end{abstract}

The European Physical Society Conference on High Energy Physics 22-29 July 2015

Vienna, Austria

${ }^{*}$ Speaker. 


\section{Introduction}

The $B_{s}^{0}$ system can provide excellent probes to test new physics in the CP violating scenarios since new phenomena beyond the SM may enter in the loop-mediated $B_{s}^{0}$ mixing process and lead to discrepancies with the SM expectations. The time evolution of $B_{S}^{0}$ meson mixing is characterised by the mass difference $\Delta m_{s}$ of the heavy $B_{H}$ and light $B_{L}$ mass eigenstates, the CP-violating mixing phase $\phi_{s}$ and the width difference $\Delta \Gamma_{s}=\Gamma_{L}-\Gamma_{H}$ of $B_{L}$ and $B_{H}$.

In the SM, the phase $\phi_{S}$ is small and can be related to CKM quark mixing matrix elements via the relation $\phi_{s} \simeq-2 \beta_{s}=-2 \arg \left[-\left(V_{t s} V_{t b}^{*}\right) /\left(V_{c s} V_{c b}^{*}\right)\right]=-0.0368 \pm 0.0018 \mathrm{rad}$, measured via global fits to experimental data [1]. However, the small uncertainty makes the direct measurement interesting since new physics could modify the phase $\phi_{s}$, if new particles enter the $B_{s}-\bar{B}_{s}$ box diagrams. Physics beyond the SM is not expected to affect $\Delta \Gamma_{s}$ as significantly as $\phi_{s}$. Nevertheless, the measurement of $\Delta \Gamma_{s}$ is useful as it allows theoretical predictions to be tested.

\section{The ATLAS Detector}

The ATLAS (A Toroidal Lhc ApparatuS) detector [2, 3] is a general purpose experiment operating at the Large Hadron Collider (LHC) at CERN, and it is divided in three main components (see Figure 1). Moving outward from the interaction point, the detector consists of a tracking system (inner detector) able to measure the directions and momenta of the charged particles. The calorimeter system measures the energies of the electrons, photons and hadrons. The muon spectrometer measures the momentum and position of the muons that have enough energy to reach it. Furthermore, a magnet system provides a magnetic field, allowing the tracker and the muon spectrometer to perform measurements of the momentum of charged particles.

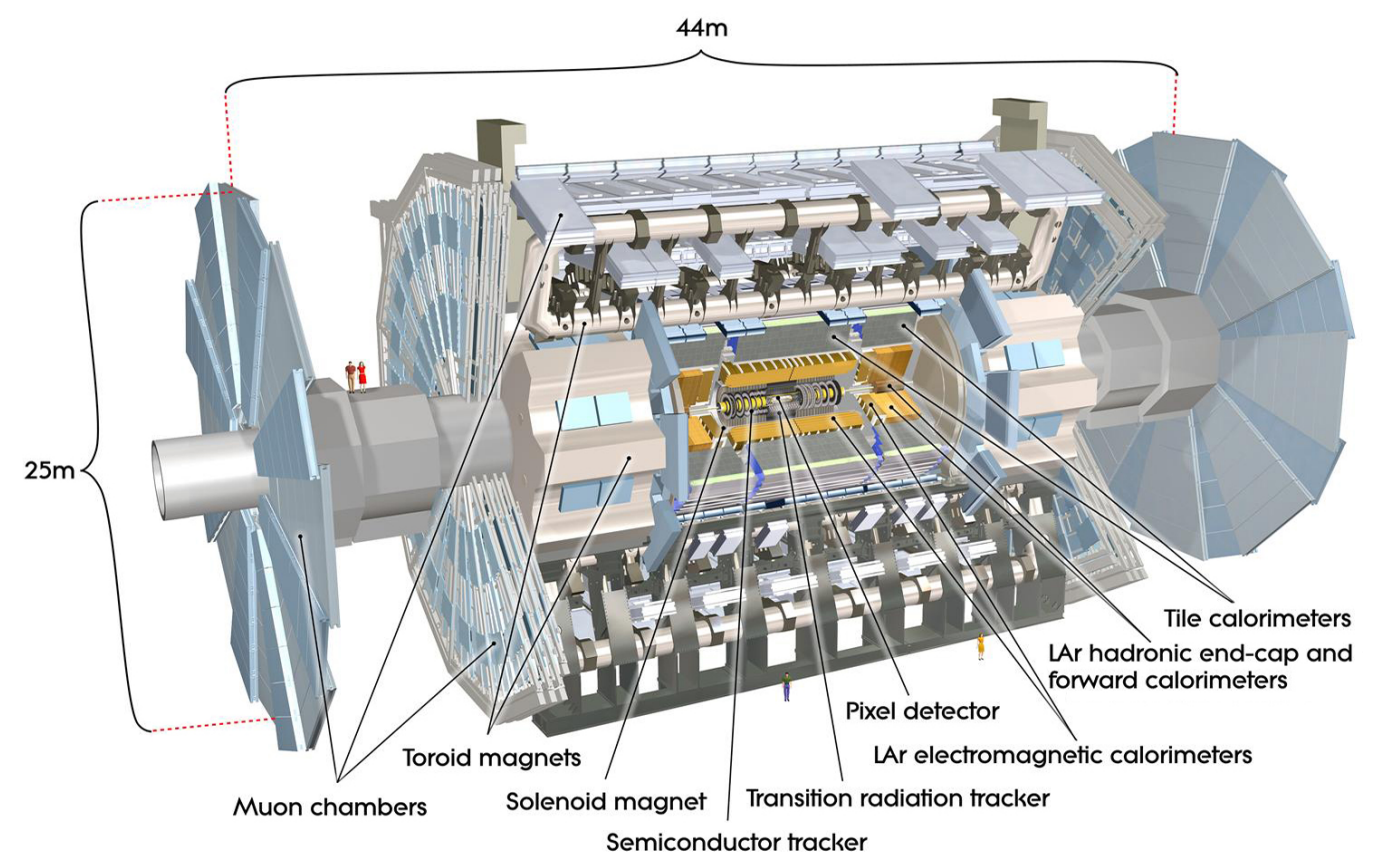

Figure 1: Overview of the ATLAS detector (taken from Ref. [2]). 
The muon and tracking systems are of particular importance in the reconstruction of B meson candidates. Only data collected when both these systems were operating correctly and where the LHC beams were declared to be stable are used in the analysis. A muon identified using a combination of muon spectrometer and inner detector track parameters is referred to as a combined-muon, whereas a muon formed from a track segment that is not associated with a muon spectrometer track but is matched to an inner detector track extrapolated to the muon spectrometer is referred to as a segment-tagged muon.

ATLAS can perform high quality measurements of the CP-violating phase in $B_{s}^{0} \rightarrow J / \psi \phi$ decay and indirect searches for new physics in rare B decays. These are complementary tools to direct searches for new physics and can provide precise measurements to compare with SM predictions and set important constraints to new physics model building.

In this document we present the measurement of the $B_{s}^{0} \rightarrow J / \psi \phi$ decay parameters obtained with $14.3 \mathrm{fb}^{-1}$ of integrated luminosity collected by the ATLAS detector during $8 \mathrm{TeV} p p$ collisions recorded in 2012 data taking period [4]. The results are statistically combined with those obtained with $4.9 \mathrm{fb}^{-1}$ of data collected in 2011 at $7 \mathrm{TeV}$.

\section{3. $B_{s}^{0} \rightarrow J / \psi \phi$ Angular Analysis}

The $B_{s}^{0} \rightarrow J / \psi \phi$ final-state is an admixture of different $\mathrm{CP}$ eigenstates (CP-odd and CP-even states with orbital angular momentum $\mathrm{L}=0,1$ or 2 ). In order to disentangle them, the timedependent angular distribution of the final state particles, described by Equation 3.1,

$$
f\left(t, \theta_{T}, \psi_{T}, \phi_{T}\right)=\sum_{k=1}^{10} \mathscr{O}^{(k)}(t) \cdot g^{(k)}\left(\theta_{T}, \psi_{T}, \phi_{T}\right),
$$

has to be performed (for more details see [5]). The functions $\mathscr{O}^{(k)}(t)$ describe the evolution of the angular distributions with the proper time of the $B_{s}^{0}$ decay and are expressed in term of the linear polarisation amplitudes $A_{0}(t), A_{\|}(t)$ and $A_{\perp}(t)$, while $g^{(k)}$ are the 10 angular functions [6]. Flavour tagging is used to distinguish between the initial $B_{s}^{0}$ and $\bar{B}_{s}^{0}$ states.
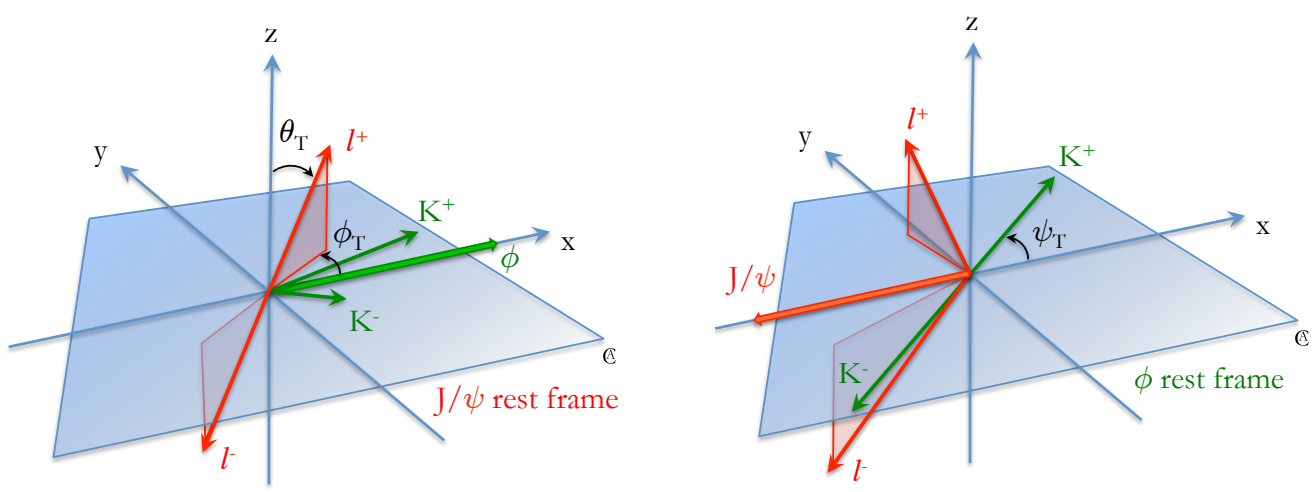

Figure 2: Schematic definition of coordinate systems and angles for the $B_{s}^{0} \rightarrow J / \psi \phi$ decay (taken from Ref. [7]).

Figure 2 illustrates the angles used in the analysis that are defined in the rest frame of the final-state particles. The $x$ axis is determined by the direction of the $\phi$ meson in the $J / \psi$ rest frame 
while the $K^{+} K^{-}$plane defines the $x y$ plane. $\theta_{T}$ is the angle between $\mu^{+}$momentum $p\left(\mu^{+}\right)$and the $x y$ plane in the $J / \psi$ meson rest frame, $\phi_{T}$ is the angle between the $x$ axis and the projection of the $\mu^{+}$momentum in the $x y$ plane $p_{x y}\left(\mu^{+}\right)$in the $J / \psi$ rest frame, finally $\psi_{T}$ is the angle between $p\left(K^{+}\right)$and $-p(J / \psi)$ in the $\phi$ meson rest frame.

\section{Flavour Tagging}

The determination of the initial flavour of the neutral $B$ meson can be inferred using the information from the other $B$ meson that is typically produced from the other $b$ quark in the event, referred as the Opposite-Side Tagging (OST). This method has been studied and calibrated on $B^{ \pm} \rightarrow J / \psi K^{ \pm}$samples in which the flavour of the charge of the B meson at production is given by the charge of the kaon.

Two different tagging methods are used in the analysis, a muon (electron) tagger in which the charge is identified through the semi-leptonic decay of the $B$ and a charge jet tagger [8] that considers tracks associated to the jet from the same primary vertex of the signal decay. The two methods are combined according to the hierarchy of performances. If it is not possible to provide a tagging response for the event, the tagging probability is set to 0.5 .

\section{Maximum Likelihood Fit}

The parameters of the $B_{s}^{0} \rightarrow J / \psi\left(\mu^{+} \mu^{-}\right) \phi\left(K^{+} K^{-}\right)$decay have been extracted by means of an un-binned maximum likelihood fit that uses the information from the reconstructed mass, the measured proper decay time, the measured proper decay time uncertainty, the tag probability and the three transversity angles (described in section 3) of each $B_{s}^{0} \rightarrow J / \psi \phi$ candidate. The background is composed of prompt and non-prompt combinatorial events, as well as $B^{0} \rightarrow J / \psi K^{* 0}$ and $B^{0} \rightarrow J / \psi K \pi$ decays. The likelihood function is defined as a combination of the signal and background probability density functions described in detail in [6].

The systematic uncertainties are estimated taking into account several effects that are not considered in the likelihood fit. The statistical uncertainties associated to the flavour tagging are due to the finite size of the $B^{ \pm} \rightarrow J / \psi K^{ \pm}$calibration samples and they are treated in the overall statistical error. The systematic uncertainty arises from the precision of the tagging calibration and it is estimated by changing the model used to parameterise the probability distribution as a function of tag charge. Systematic uncertainties may come from the assumption of the fit model, and these effects have been estimated by means of pseudo-experiments varying the model tested. Trigger efficiency and background angles model have also been considered in the systematic as well as the contamination from $B^{0} \rightarrow J / \psi K^{* 0}$ and $B^{0} \rightarrow J / \psi K \pi$ events mis-reconstructed as $B_{s}^{0} \rightarrow J / \psi \phi$. Table 1 reports the total systematic uncertainty assigned to the physical parameters.

Table 1: Summary of the systematic uncertainties assigned to the physical parameters (taken from Ref. [4]).

\begin{tabular}{c|ccccccccc}
\hline & $\phi_{s}$ & $\Delta \Gamma_{s}$ & $\Gamma_{s}$ & $\left|A_{\|}(0)\right|^{2}$ & $\left|A_{0}(0)\right|^{2}$ & $\left|A_{S}(0)\right|^{2}$ & $\delta_{\perp}$ & $\delta_{\|}$ & $\delta_{\perp}-\delta_{S}$ \\
\hline Total & 0.026 & 0.005 & 0.003 & 0.006 & 0.001 & 0.007 & 0.241 & 0.037 & 0.031 \\
\hline
\end{tabular}




\section{2012 Results}

An un-binned maximum likelihood fit including a tagging probability is performed using 14.3 $\mathrm{fb}^{-1}$ of data collected in 2012 data taking period and the results obtained for the nine measured physical parameters are reported in Table 2 .

Table 2: Fitted values for the physical parameters along with their statistical and systematic uncertainties obtained using 2012 data (taken from Ref. [4]).

\begin{tabular}{cccc}
\hline Parameter & Value & Statistical uncertainty & Systematic uncertainty \\
\hline$\phi_{S}(\mathrm{rad})$ & -0.097 & 0.077 & 0.026 \\
$\Delta \Gamma_{S}\left(\mathrm{ps}^{-1}\right)$ & 0.109 & 0.014 & 0.005 \\
$\Gamma_{S}\left(\mathrm{ps}^{-1}\right)$ & 0.687 & 0.004 & 0.003 \\
$\left|A_{\|}(0)\right|^{2}$ & 0.231 & 0.005 & 0.006 \\
$\left|A_{0}(0)\right|^{2}$ & 0.516 & 0.004 & 0.001 \\
$\left|A_{S}(0)\right|^{2}$ & 0.103 & 0.008 & 0.007 \\
$\delta_{\perp}$ & 4.481 & 0.458 & 0.241 \\
$\delta_{\|}$ & 3.151 & 0.094 & 0.037 \\
$\delta_{\perp}-\delta_{S}$ & -0.081 & 0.033 & 0.031 \\
\hline
\end{tabular}

The measured values are consistent with those obtained in the previous analysis using $4.9 \mathrm{fb}^{-1}$ of data collected in 2011 [6]. This consistency is also clear in the left plot reported in Figure 3 where the 2011 and 2012 likelihood contours in the $\phi_{s}-\Delta \Gamma_{s}$ projection are shown.

\section{Combination of 2011 and 2012 Results}

The results obtained using 2012 data are combined with those obtained in the previous analysis using a Best Linear Unbiased Estimate (BLUE) combination [9] to give an overall result for Run 1. The combined results for the fit parameters and their statistical and systematic uncertainties are summarised in Table 3, while the likelihood contours in the $\phi_{s}-\Delta \Gamma_{s}$ plane are reported in the right plot in Figure 3.

Table 3: Fitted values for the physical parameters along with their statistical and systematic uncertainties obtained from the combination of 2011 and 2012 results (taken from Ref. [4]).

\begin{tabular}{cccc}
\hline Parameter & Value & Statistical uncertainty & Systematic uncertainty \\
\hline$\phi_{S}(\mathrm{rad})$ & -0.097 & 0.077 & 0.026 \\
$\Delta \Gamma_{S}\left(\mathrm{ps}^{-1}\right)$ & 0.109 & 0.014 & 0.005 \\
$\Gamma_{S}\left(\mathrm{ps}^{-1}\right)$ & 0.687 & 0.004 & 0.003 \\
$\left|A_{\|}(0)\right|^{2}$ & 0.231 & 0.005 & 0.006 \\
$\left|A_{0}(0)\right|^{2}$ & 0.516 & 0.004 & 0.001 \\
$\left|A_{S}(0)\right|^{2}$ & 0.103 & 0.008 & 0.007 \\
$\delta_{\perp}$ & 4.481 & 0.458 & 0.241 \\
$\delta_{\|}$ & 3.151 & 0.094 & 0.037 \\
$\delta_{\perp}-\delta_{S}$ & -0.081 & 0.033 & 0.031 \\
\hline
\end{tabular}



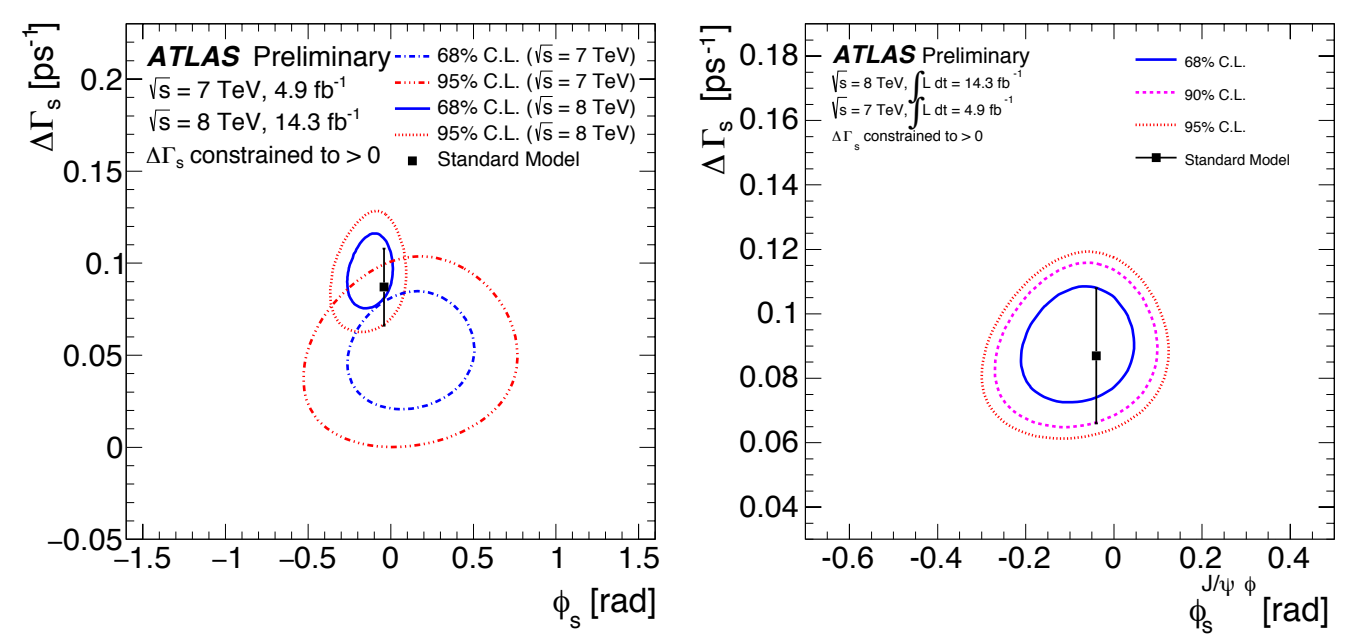

Figure 3: Likelihood contours in $\phi_{s}-\Delta \Gamma_{s}$ plane (taken from Ref. [4]) for individual results from 2011 and 2012 data (left) and a final statistical combination of the two measurements (right). The blue, pink and red lines show the likelihood contours for $68 \%, 90 \%$ and $95 \%$ confidence intervals respectively (statistical errors only).

Figure 4 reports the comparison and the combination of the ATLAS results with the results obtained by other experiments [10]. The ATLAS Run 1 measurements are compatible with the SM predictions and, within the uncertainties, they are consistent with the LHCb Run 1 results [11] for $B_{s}^{0} \rightarrow J / \psi \phi$ and $B_{s}^{0} \rightarrow J / \psi K^{+} K^{-}$decays and the CMS Run 1 results for $B_{s}^{0} \rightarrow J / \psi \phi$ [12]. LHCb has also combined its results with the measurements for $B_{s}^{0} \rightarrow J / \psi \pi^{+} \pi^{-}$that increased the precision on $\phi_{s}$.

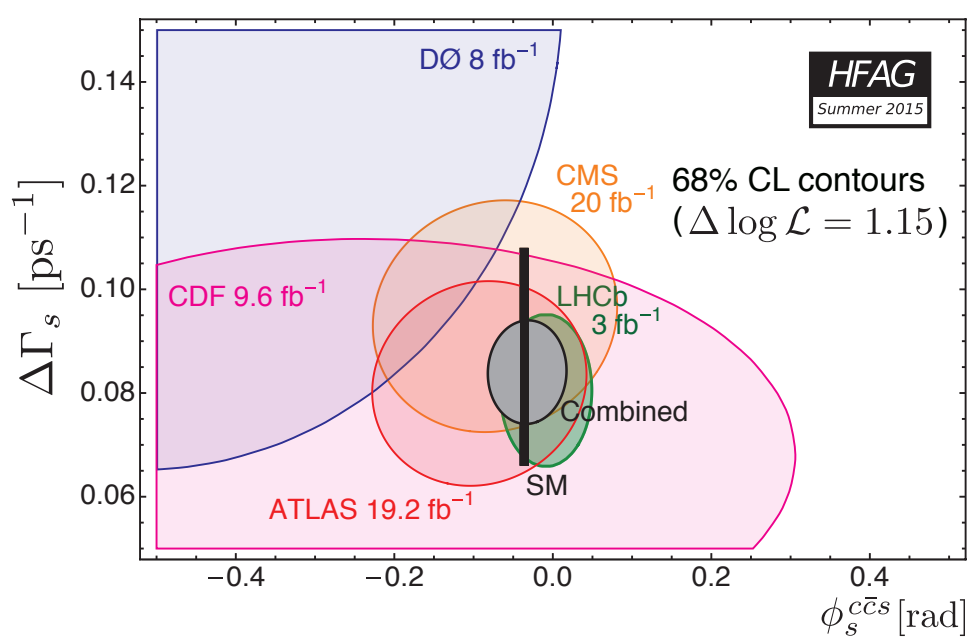

Figure 4: Heavy Flavor Averaging Group (HFAG) comparison and combination of the ATLAS results with the results of other experiments (taken from Ref. [10]). The vertical black line represents the SM expectation. 


\section{References}

[1] UTfit Collaboration, M. Bona et al., emphConstraints on New Physics from the Quark Mixing Unitarity Triangle, Phys. Rev. Lett. 972006151803.

[2] ATLAS Collaboration, The ATLAS Experiment at the CERN Large Hadron Collider, JINST 32008 S08003.

[3] ATLAS Collaboration, The ATLAS Inner Detector commissioning and calibration, Eur. Phys. J. C70 (2010), 787 - 821. arXiv:1004.5293.

[4] ATLAS Collaboration, to be published.

[5] P. Ball et al., B decays at the LHC CERN-TH/2000-101, arXiv:hep-ph/0003238.

[6] ATLAS Collaboration, Time-dependent angular analysis of the decay $B_{s}^{0} \rightarrow J / \psi \phi$ and extraction of $\Delta \Gamma_{s}$ and the CP-violating weak phase $\phi_{S}$ by ATLAS, JHEP 1212 (2012) 072, arXiv:1208.0572 [hep-ex].

[7] Cristiano Alpigiani, Testing the new physics scale in the $B^{0}$ system at ATLAS, Il Nuovo Cimento, Vol. 37 C, N. 6.

[8] ATLAS Collaboration, Expected Performance of the ATLAS Experiment - Detector, Trigger and Physics, arXiv:0901.0512.

[9] R. Nisius, On the combination of correlated estimates of a physics observable, The European Physical Journal C 74 (2014) no. 8, 3004.

[10] Y. Amhis et al., Heavy Flavour Averaging Group (HFAG), http://lepbosc.web.cern.ch/LEPBOSC/combined_results/summer_2015/\#DG.

[11] LHCb Collaboration, Precision measurement of $C P$ violation in $B_{s}^{0} \rightarrow J / \psi K^{+} K^{-}$decays, Phys. Rev. Lett. 114 (2015) 041801, arXiv:1411.3104 [hep-ex].

[12] CMS Collaboration, Measurement of the CP-violating weak phase phi-s and the decay width difference DeltaGamma-s using the Bs to J/psiPhi(1020) decay channel, Tech. Rep.

CMS-PAS-BPH-13-012, CERN, Geneva, 2014. 